\title{
Profile of Anti-Tp47 Antibodies in Patients With Positive Serology for Syphilis Analized by Western Blot
}

\author{
Ana Paula Félix de Miranda and Neuza Satomi Sato \\ Section of Serology, Division of Medical Biology, Adolfo Lutz Institute; São Paulo, SP, Brazil
}

\begin{abstract}
In Brazil, syphilis is still a great problem of public health. Serological test is essential for syphilis diagnosis and the current trend is the use of recombinant antigen in the treponemal tests, due to its confirmed higher sensibility and specificity. The purpose of the present study was to analyze the profile of anti-Tp47 antibodies in patients with positive serology for syphilis. One hundred positive sera samples were analyzed by Western Blot (WB) technique, using the recombinant antigen (rTp47). Ten of them did not present antibodies against the fraction rTp47, the results were confirmed by WB using native T. pallidum antigen. All ten samples had antibodies against the fractions Tp17 and Tp15 and presented low reactivity in VDRL, negative results or title below than 1:4. Considering that VDRL is used for therapeutic monitoring due to seroreversion of nontreponemal antibodies in response to the treatment, and that some studies reported loss of treponemal antibodies after treatment, we could speculate if these ten samples are cases of serological memory from patients previously treated for syphilis. In addition, although several features state the Tp47 fraction as one of the major antigenic components, based on our results we point out to the importance of including other antigenic proteins such as Tp17 and Tp15 in addition to Tp47 in tests for serological screening of syphilis.
\end{abstract}

Key-Words: Syphilis, Treponema pallidum, recombinant antigens, serological test.

Syphilis is one of the most prevalent sexually transmitted disease; it is caused by the spirochete Treponema pallidum subsp. pallidum. Although effective therapy has been available for more than four decades, the disease continues to be a global public health problem. The World Health Organization estimates that there are approximately 12 million new cases of syphilis in adults worldwide [1]. In Brazil, the prevalence of syphilis was $0.874 \%$ in a study conduced in 2002 with male population group with age between 19 and 21 . In another study with pregnants aging between 15 and 49, the prevalence of active syphilis was $1.6 \%$. At present, there is a great governmental effort for eradication of vertical transmission of syphilis in Brazil [2].

The infection is transmitted through sexual contact or vertically during pregnancy with various manifestations that change according to the duration of illness. Clinically, syphilis is classified into several stages that follow each one temporally in untreated patients (primary, secondary, latent and tertiary). These stages have implications regarding diagnosis and treatment of syphilis [3].

Laboratorial diagnosis of syphilis is crucial to the epidemiological and diagnostic evaluation of the disease. The detection of the etiologic agent is cumbersome, because the direct detection of this spirochete is applicable only in skin lesion (primary and secondary stages), and the sensibility is low. Furthermore, the T. pallidum can not be cultivated in vitro and molecular techniques are not available for routine

Received on 28 November 2007; revised 3 April 2008.

Address for correspondence: Dr. Neuza Sato. Adolfo Lutz Institute. Av. Dr. Arnaldo, 355, 10th floor. Zip code: 1246-902 - São Paulo, SP, Brazil. Phone.: 55-11-3068-2885, fax: 55-11-3085-3505. E-mail: neuzasat@uol.com.br.

The Brazilian Journal of Infectious Diseases 2008;12(2):139-143. (C) 2008 by The Brazilian Journal of Infectious Diseases and Contexto Publishing. All rights reserved. use. Therefore, the laboratorial diagnosis is performed mainly by serological tests, which are divided into nontreponemal and treponemal tests. The nontreponemal tests include the Venereal Disease Research Laboratory (VDRL) which is useful for screening and to evaluate the response to treatments. Treponemal tests are used to confirm the diagnosis and include hemagglutination assay (TPHA), fluorescent treponemal antibody absorption (FTA-ABS) and enzyme-linked immunosorbent assay (ELISA) and detect specific antitreponemal antibodies.

Formerly, several ELISA based on whole cell lysate were developed and they presented sensibility of $93.3 \%$ to $100 \%$ and specificity of $95.5 \%$ to $99.8 \%$ [4-7]. In recent years, as well as in other diseases, the trend is to use the recombinant antigens, mainly the fractions of $47 \mathrm{kDa}, 17 \mathrm{KDa}$ and $15 \mathrm{kDa}$. These three antigens are membrane-integrated proteins. The Tp47 is the most abundant and highly immunogenic [8-10], while the 15 and $17 \mathrm{kDa}$ are present in lower amount, although they both are strongly immunogenic [11,12]. Recombinant antigen-based ELISA, using these fractions, showed diagnostic performance similar or superior to the classic treponemal tests; many of them are commercially available [13-18].

The Tp47 fraction is one of the major antigenic components due to several features: $i$ ) it is a reliable immunological marker for human syphilis infections [19-21]; ii) it triggers an early humoral response, three to six days after infection, as observed in a study of ontogeny of humoral immunity in experimental syphilis [22]; iii) anti-Tp47 antibodies are present in all stages of non-treated syphilis [23]; iv) the Tp47 antigen had been reported as a potential marker for active syphilis disease, as anti-Tp47 IgM had been detected in patients with congenital syphilis, by WB assay [21]; v) the decrease of anti-Tp47 in response to treatment allowed the suggestion of this fraction as a candidate for therapeutic marker [24]. 
The present study aimed to analyze the reactivity profile of anti-Tp47 in serum samples from patients with laboratorial diagnosis of syphilis.

\section{Material and Methods}

\section{Sera Samples}

A total of 200 sera samples previously tested by VDRL and TPHA or FTA-ABS were obtained from the Laboratory of Serology at Adolfo Lutz Institute: 100 samples with negative and 100 with positive results for serological tests, all of the latter with positive treponemal tests and 84 with positive results for VDRL.

\section{Antigens}

Recombinant rTp47, the recombinant antigen rTp47 produced in fusing with GST and purified by affinity chromatography was used [25]; native antigen of T. pallidum, soluble antigen of $T$. pallidum, Nichols strain, prepared by extraction with detergent Zwittergent ${ }^{\circledR}$ with protein concentration of $0.57 \mathrm{mg} / \mathrm{mL}$, kindly provided by Wama Diagnostica.

\section{ELISA-rTp47}

An rTp47-based ELISA was used for initial screening. Eight-well strip plates (High Binding 1, Costar 2592) were coated overnight at $4^{\circ} \mathrm{C}$ with $100 \mu \mathrm{L}$ of rTp47 (49 ng/well) in phosphate buffered saline (PBS), $\mathrm{pH} 7.2,0.15 \mathrm{M}$. Plates were washed three times with $\mathrm{NaCl} 0.15 \mathrm{M}, 0.05 \%$ Tween 20 , five minutes each. Human sera were diluted to 1:21 in sample buffer (PBS pH 7.2, 0.15M, 2.5\% sodium caseinate) and $100 \mu \mathrm{L}$ was added to the well and incubated for $1 \mathrm{~h}$ at $37^{\circ} \mathrm{C}$. After washing, $100 \mu \mathrm{L}$ of a 1:5000 diluted goat anti-human IgG alkaline phosphatase (Genelabs) were applied and incubated at $37^{\circ} \mathrm{C}$ for $1 \mathrm{~h}$. Plates were developed for $20 \mathrm{~min}$ at $37^{\circ} \mathrm{C}$ with $100 \mu \mathrm{L}$ of p-nitrophenyl phosphate (pNPP) per well, and the absorbance at $405 \mathrm{~nm}$ was measured. A weakly reactive control serum was added in triplicate on each plate and its absorbance value was defined as the cut-off, which was equivalent to the mean plus three times the standard deviation of the absorbance of the negative control serum.

\section{Western Blot (WB), WB-rTp47 and WB-Tp Native}

It was made a sodium dodecyl sulphate-polyacrylamide gel electrophoresis (SDS-PAGE) with recombinant antigen and other with native antigen. The gel was electrophoresed using a discontinuous buffer system [26] in a minigel system, at 100 volts constant power. Low molecular weight standards and prestained low molecular weight markers (Bio-Rad Laboratories, Richmond, Calif., USA) were run with each gel to determine efficacy of electrophoretic transfer. The separated proteins were blotted onto nitrocellulose sheets $(0.2 \mu \mathrm{m})$ (Sigma) in a Semi-Dry Transfer Unit (Bio-Rad Laboratories, Richmond, Calif., USA), at 100 volts constant power for 60 minutes [27]. Following transfer, the membrane was blocked in a 5\% solution of powdered nonfat milk in Tris-saline for one hour, rinsed three times in Tris-saline and cut into $5 \mathrm{~mm}$ strips. The strips were used immediately or allowed to dry and stored at $4^{\circ} \mathrm{C}$.

All the following stages were performed at room temperature with rocking. Strips for immunoblotting were placed in individual troughs in blotting trays and incubated overnight with sera at dilution of $1: 100$ in $5 \%$ solution of powdered nonfat milk in Tris-saline/Tween 20. The strips were washed three times with Tris-saline/Tw (five minutes each) and incubated for 30 minutes in horseradish peroxidaseconjugated goat anti-human IgG at dilution of 1:1000 (Genelabs, Geneva, Switzerland). The strips were washed as described previously and developed with a 4-chloro-1-naphthol peroxidase substrate system for eight minutes. The reaction was blocked with distilled water. The strips had been left for ten minutes at $37^{\circ} \mathrm{C}$ for drying and kept under the cover of the light for analysis of the results.

\section{Serological Tests for Syphilis}

VDRL (Wiener, Rosario, Argentine); TPHA (Wama Diagnóstica, São Carlos, SP, Brazil); FTA-ABS (Wama Diagnóstica, São Carlos, SP, Brazil); ELISA (ICE Syphilis, Abbott-Murex, United Kingdom). The assays had been carried through in accordance with the respective procedures techniques recommended by the manufacturer.

\section{Results \\ ELISA-rTp47}

In the preliminary analysis of 200 serum samples by ELISArTp47, which had an agreement with other treponemal tests (FTA-ABS and TPHA) of 92\%, presenting the co-positivity and co-negativity, respectively of $85.5 \%$ and $98 \%$. Two samples with false-positive results in ELISA-rTp47 presented low reactivity; the cut-off indexes were 1.08 and 1.29. Fourteen samples had false-negative results in ELISA-rTp47; however four showed reactivity against Tp47 fraction in WB.

\section{Western Blots}

A total of 102 samples were analyzed by WB-rTp47; two samples with false-positive results in ELISA-rTp47 were negative in WB-rTp47 and 90\% of 100 positive samples showed reactivity against $\mathrm{Tp} 47$ fraction. Ten sera with negative WBrTp47 were further tested by WB using native antigen of $T$. pallidum and no antibodies against Tp47 antigen, but they presented strong reactivity with Tp17/15 protein. The WB reactivity profile is presented in Figure 1, recombinant antigen (1A) and native antigen (1B). The position of Tp47, Tp17 and Tp15 in the WB native Tp was previously verified in reaction performed with affinity purified monospecific antibodies. In this assay, the proteins Tp17 and Tp15 were overlap in the same position.

Other Serological Tests for Syphilis

The results of serological tests (WB-rTp47, WB-Tp native, 
ELISA, FTA-ABS, TPHA and VDRL) performed with these ten samples are presented in Table 1 . When submitted to ELISA (ICE Syphilis, Abbott-Murex, United Kingdom), three of them (samples 2, 3 and 8) showed reactivity around 20\% of cut-off value and seven samples presented strong positive reaction with cut-off index ratio varying between 2.07 and 10.87. The higher level reaction observed should be due to antibodies against rTp17 and rTp15, as this ELISA is based in three recombinants antigens rTp47, rTp17 and rTp15.
Comparison of WB r-Tp47 and VDRL Reactivity in Serum Specimen

The VDRL results in these 100 samples showed the following distribution: VDRL was non reactive in 16\%, while $7 \%$ had positivity at dilution $1: 1$, the most of them (60\%) was positive in dilution ranged from $1: 2$ to $1: 16$, and $11 \%$ presented positive reaction from 1:32 to 1:256. Curiously, all samples without detectable antibodies against rTp47 antigen showed low reactivity in VDRL, with title below 1:4 and in 40\% VDRL was negative (Figure 2).

Figure 1. Profile of reactivity in the WB with recombinant antigen rTp47 (left) and native antigen (right), reaction carried through with the 1/100 diluted serum and revelation using the system alkaline phosphates and BCIP/NBT. Positive (1) and negative (2) controls; and clinical samples (3-6).
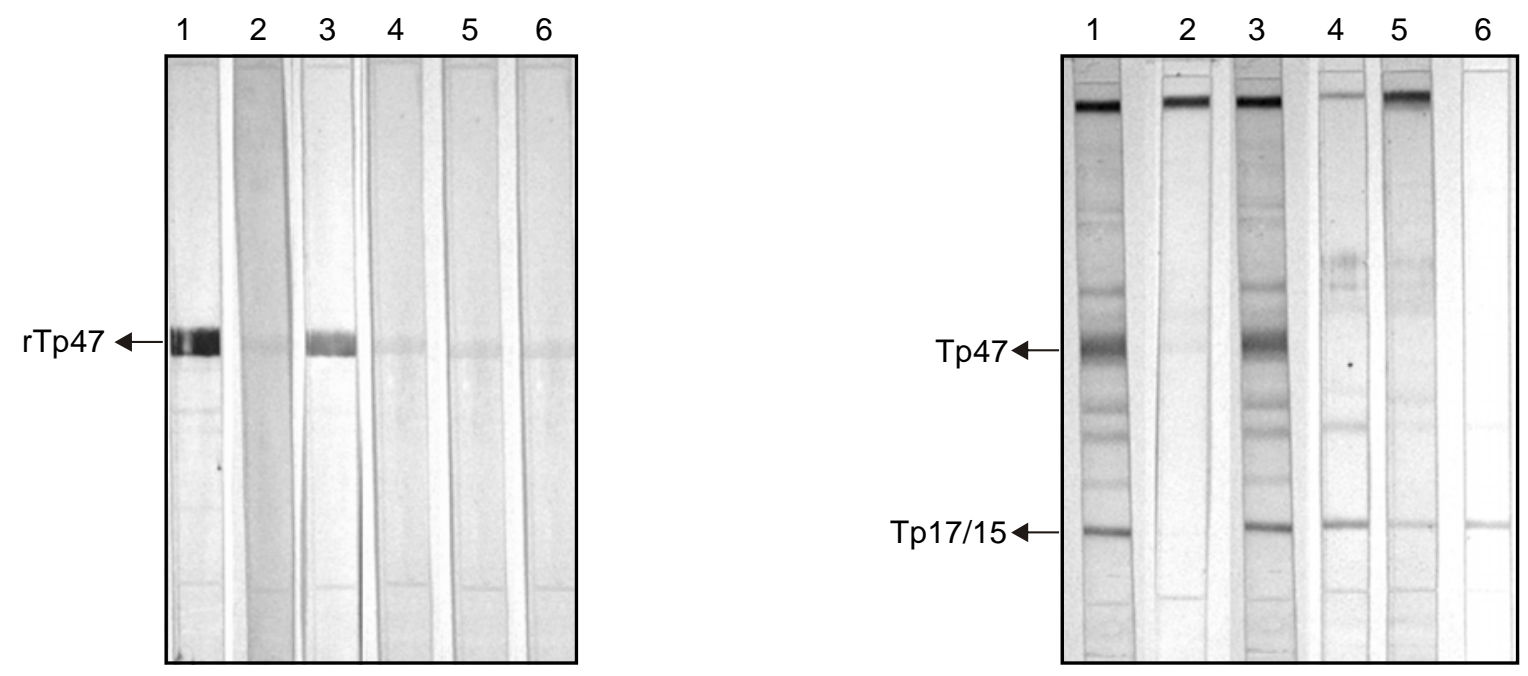

Table 1. Results of ten samples without antibodies against the Tp47 antigen and of the positive (CP) and negative (CN) controls, in the tests of WB-rTp47, WB-rTp native, ELISA Murex, FTA-ABS, TPHA and VDRL

\begin{tabular}{|c|c|c|c|c|c|c|}
\hline $\begin{array}{c}\text { Sample } \\
\text { Nr. }\end{array}$ & WB-rTP47 & $\begin{array}{c}\text { WB-Tp } \\
\text { native } \\
\text { Tp17/15 }\end{array}$ & $\begin{array}{l}\text { ELISA-MUREX } \\
\text { (I-CO) }\end{array}$ & FTA-Abs & TPHA & VDRL \\
\hline 1 & - & + & 2.07 & + & + & $1 / 2$ \\
\hline 2 & - & + & 1.16 & + & + & - \\
\hline 3 & - & + & 1.20 & + & + & - \\
\hline 4 & - & + & 10.87 & + & $+/-$ & $1 / 4$ \\
\hline 5 & - & + & 4.67 & + & - & $1 / 2$ \\
\hline 6 & - & + & 8.19 & + & + & $1 / 2$ \\
\hline 7 & - & + & 1.96 & + & + & $1 / 4$ \\
\hline 8 & - & + & 1.02 & + & + & $1 / 1$ \\
\hline 9 & - & + & 9.55 & + & + & - \\
\hline 10 & - & + & 9.60 & + & - & - \\
\hline PC & + & + & 10.61 & + & + & $1 / 8$ \\
\hline NC & - & - & 0.35 & - & - & - \\
\hline
\end{tabular}


Figure 2. Analysis of 100 samples with positive serology for syphilis in accordance with the reactivity in the VDRL test. Number of cases with and without antibodies against the antigen rTp47 distributed in accordance with the VDRL title.

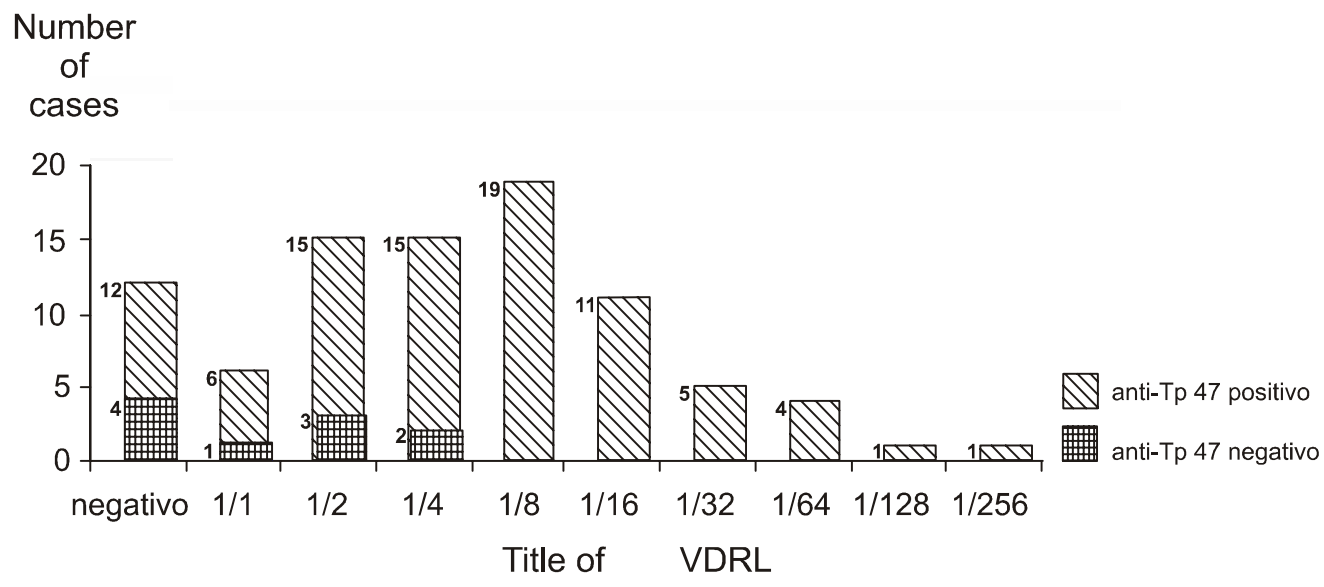

\section{Discussion}

The object of the present study was to analyze anti-rTp47 antibodies profile in patients with positive results in serological tests for syphilis. The results were unexpected, where the antibodies against fraction rTp 47 were not detected in $10 \%$ of positive samples. These sera showed reaction against other fraction of T.pallidum, mainly Tp17 and Tp15 as observed in WB performed with native antigen of T. pallidum and in ELISA (ICE Syphilis, Abbott-Murex), which is based on three recombinant antigen (47, 17 and $15 \mathrm{kDa}$ ).

There is no established correlation between VDRL and treponemal test. Usually, VDRL title declines in response to treatment while anti-treponemal antibodies persist as serological memories for longer period of time. So, nontreponemal test is used for follow-up of syphilis treatment, however seroreversion depends on the stage of disease, the reactive antibodies level before the start of treatment as well as the dosage of antibiotic used. It has been reported that seroreversion is more rapid after therapy if the duration of infection or clinical lesions is short and the initial nontreponemal titer is low. In patients with previous syphilis who became re-infected or in patients with late syphilis, the serological response is often slower [28,29].

A few studies are reported concerning treponemal antibodies profiles in serological response to treatment of syphilis. One study was carried out by Romanowski et al.; they observed that treponemal antibodies level also decreases in response to treatment. There was a positive correlation between the treatment introduction and the duration of treponemal antibodies memories, with early decrease of treponemal antibodies in prime-infection rather than in re-infection [30]. Other studies were performed by Western Blot technique, which provides analyses of reactivity against each specific antigenic fraction. Kim et al. observed a significant loss of anti-Tp47 after treatment of primary syphilis, with complete seroreversion in 11 months [24]. George group studied 124 persons with clinically diagnosed syphilis by using densitometric quantization and spreadsheet normalization to refine the parameters defining treponemal WB for syphilis. The reactivity against Tp47 was $100 \%$ before treatment, while $28 \%$ had lost anti-Tp47 over 12 months after treatment [31].

In the present study, $16 \%$ of positive samples had negative results for VDRL suggesting a serorevertion in response to treatment. However, the precise interpretation was limited, since no clinical or epidemiological data was available for these samples. Our results showed that $10 \%$ of positive samples had undetectable anti-Tp47. Furthermore, all ten sera had low title VDRL $(<1: 4)$ including four samples with negative results for VDRL.

Considering all together we could speculate if these ten samples are a serofast reaction from patients precociously treated. In order to clear this hypothesis more detailed, studies should be carried out including samples of treatment followup from patients with complete clinical information.

Finally, although several features state the Tp47 fraction as one of the major antigenic components, based on these results we point out to the importance of including other antigenic proteins such as Tp17 and Tp15 in addition to Tp47 in tests for serological screening of syphilis.

\section{Acknowledgment}

We are grateful to syphilis lab. members Elaine L. Oliveira and Edilene R. P. Silveira for valuable discussions. We would like to thank Wama Diagnóstica for gentle donation of $T$. pallidum soluble antigen and to Dr. Antônio M. Levy for critical comments of this manuscript. APFM is the recipient of a fellowship from Adolfo Lutz Institute supported by Fundação de Desenvolvimento Administrativo (FUNDAP).

\section{References}

1. World Health Organization. Global prevalence and incidence of selected curable sexually transmitted infections: overview and estimates 2001;02. 
2. Epidemiology. Health Ministry of Brazil [serial on the internet], 2007; [about 1 p.] Available from: http://www.aids.gov.br/data/ Pages/LUMISBCD47A0DPTBRIE.htm

3. Center for Disease Control (CDC). Case definitions for infectious condition under Public Health surveillance. Morbid. Mortal. Wkly Rep 1997;46:37-8.

4. Young H., Moyes A., McMillan A., Robertson D.H. Screening for treponemal infection by new enzyme immunoassay. Genitourin Med 1989;65:72-8.

5. Lefevre J.C., Bertrand M.A., Laring M.B. False positive reactions occurring with the Captia Syphilis G EIA in sera from patients with Lyme disease. Genitourin Med 1992;68:142.

6. Pope V., Fears M.B., Morill W.E., et al. Comparassion of the Serodia Treponema pallidum particle agglutination, Captia Syphilis G and Spirotek Reagin II testes with standard tests techniques for diagnosis of syphilis. J Clin Microbiol 2001;39:3817.

7. Castro R., Prieto E.S., Santo I., et al. Evaluation of an enzyme immunoassay technique for detection of antibodies against Treponema pallidum. J Clin Microbiol 2003;41:250-3.

8. Norgard M.V., Chamberlain N.R., Swanaitt M.A., Goldberg M.S. Cloning and Expression of the major 47 kilodalton surface immunogen of Treponema pallidum in Escherichia coli. Infect Immun 1986;54:500-6.

9. Chamberlain N.R., Brant M.E., Erwuin A.L., et al. Major integral membrane protein immunogens of Treponema pallidum are proteolipids. Infect Immun 1989;57:2872-7.

10. Weigel L.M., Brant M.E., Nogard M.V. Analysis of N-terminal region of the 47-kilodalton integral membrane lipoprotein of Treponema pallidum. Infect Immun 1992;60:1568-76.

11. Purcell B.K., Chamberlain N.R., Goldberg M.S., et al. Molecular cloning and characterization of the 15-kilodalton major immunogen of Treponema pallidum. Infect Immnun 1989;57:3708-14.

12. Akins D.R., Purcell B.K., Mitra M.M., et al. Lipid modification of the 17-kilodalton membrane immunogen of Treponema pallidum determines macrophage activation as well as amphiphilicity. Infect Immun 1993; $61: 1202-10$.

13. Fujimura K., Ise N., Ueno E., et al. Reactivity of recombinant Treponema pallidum ( $\mathrm{r}-\mathrm{Tp}$ ) antigens with anti-Tp antibodies in human syphilitic sera evaluated by ELISA. J Clin Lab Anal 1997;6:315-22.

14. Medicines and Healthcare Products Regulatory Agency. Evaluation MHRA 04109 Ten Syphilis EIAs in London, UK, 2004. 47p.

15. Sambri V., Marangoni A., Simone M.A., et al. Evaluation of recomwell Treponema, a novel recombinant antigen basead enzyme linked immunosorbent assay for the diagnosis of syphilis. Clin Microbiol infect 2001;7:200-5.
16. Rodriguez I., Alvarez E.L., Fernandes C., Miranda A. Comparassion of a recombinant antigen immunoassay with Treponema pallidum hemagglutination test for serological confirmation of syphilis. Mem Inst Oswaldo Cruz 2002;97:347-9.

17. Schmidt B.L. Evaluation of a new particle gel immunoassay for determination of antibodies against Treponema pallidum. J Clin Microbiol 2004;42:2833-5.

18. Aktas G., Young H., Moyes A., Bader S. Evaluation of the Serodia Treponema pallidum particle agglutination, the Murex Syphilis ICE and the Enzywell TP testes for serodiagnosis of syphilis. Int J STD AIDS 2005; $16: 294-8$.

19. Hanff P.A., Fehninger T.E., Miller J.N., Lovett M.A. Humoral immune response in human syphilis to polypeptides of Treponema pallidum. J Immunol 1982;129:1287-91.

20. Hensel V., Wellensiek H.J., Bhakdi S. Sodium dodecyl sulfatepolyacrilamide gel eletrophoresis immunoblotting as a serologial tool in the diagnosis of infections. J Clin Microbiol 1985;21:82-7.

21. Lewis L.L., Taber L.H., Baughn R.E. Evaluation of immunoglobulin $\mathrm{M}$ Western Blot analysis in the diagnosis of congenital syphilis. J Clin Microbiol 1990;28:296-302.

22. Hanff P.A., Bishop N.H., Miller J.N., Lovett M.A. Humoral immune response in experimental syphilis to polypeptides of Treponema pallidum. J Immunol 1983;131:1973-7.

23. Lemos E.A., Belém Z.R., Santos A., Ferreira A.W. Characterization of the Western blotting IgG reactivity patterns in the clinical phases of acquired syphilis. Diagn Microbiol Infect Dis 2007;2:177-83.

24. Kim D.K., Lee M.G., Lee J.B. Changes of serum IgG antibody reactivity to protein antigens of Treponema pallidum in syphilis patients after treatment. J Korean Med Sci 1989;2:36-69.

25. Sato N.S., Hirata M.H., Hirata R.D., et al. Analysis of Treponema pallidum recombinant antigens for diagnosis of syphilis by Western Blotting technique. Rev Inst Med Trop 1999;41(2):115-8.

26. Laemmli U.K. Cleavage of structural proteins during the assembly of the head of bacteriophage T4. Nature 1970;5259:680-5.

27. Towbin H., Staehlin T., Gordon J. Eletroforetic transfer of proteins from polyacrilamide gels to nitrocellulose sheets: procedure and some applications. Proc Natl Acad Sci 1979;76:4350-4.

28. Brown S.T., Zaidi A., Larsen, S.A., Reynolds G.H. Serological response to syphilis treatment. A new analysis of old data. JAMA 1985; 9:1296-9.

29. Talwar S., Tutakne M.A., Tiwari V.D. VDRL titres in early syphilis before and after treatment. Genitourin Med 1992;2:120-2.

30. Romanowski B., Sutherland R., Fick G.H., et al. Serologic response to treatment of infectious syphilis. Ann Intern Med 1991; $114: 1005-9$.

31. George R., Pope V., Fears M., et al. An analysis of the value of some antigen-antibody interactions used as diagnostic indicators in a treponemal Western blot (TWB) test for syphilis. J Clin Lab Immunol 1998;1:27-44. 